\title{
Adalimumab-induced Anti-neutrophilic Cytoplasmic Antibody Vasculitis: A Rare Complication of an Increasingly Common Treatment
}

Tanya Chandra ${ }^{1}$, Joy-Ann Tabanor-Gayle ${ }^{2}$, Santhanam Lakshminarayanan ${ }^{2}$

1. Internal Medicine, University of Connecticut Health Center, Farmington, USA 2. Rheumatology, University of Connecticut Health Center, Farmington, USA

Corresponding author: Tanya Chandra, chandra@uchc.edu

\begin{abstract}
Tumor necrosis factor (TNF) inhibitors are used for treatment of different autoimmune diseases. Interestingly they are also being noted to cause autoimmune side effects such as vasculitis, systemic lupus erythematosus, interstitial lung disease, etc. We describe one such case of granuloma annulare being treated with Adalimumab, who then developed pulmonary-renal syndrome form anti-neutrophilic cytoplasmic antibody (ANCA)-induced vasculitis. This was treated with steroids and immunosuppression, as well as discontinuation of the TNF inhibitor. However, he remains dependant on dialysis and

immunosuppression. In this article, we review the existing literature on clinical presentation and course of TNF inhibitors-induced ANCA vasculitis. We also discuss the underlying mechanisms thought to be responsible for this.
\end{abstract}

Received 06/28/2019

Review began 08/08/2019 Review ended 09/04/2019 Published 09/09/2019

(c) Copyright 2019

Chandra et al. This is an open access article distributed under the terms of the Creative Commons Attribution License CC-BY 3.0., which permits unrestricted use, distribution, and reproduction in any medium, provided the original author and source are credited.
Categories: Internal Medicine, Allergy/Immunology, Rheumatology

Keywords: tnf inhibitor, anca vasculitis, adverse drug reactions

\section{Introduction}

Tumor necrosis factor (TNF) inhibitors are utilized for the treatment of a variety of autoimmune diseases, but have also been associated with the paradoxical emergence of autoimmune phenomena, including cutaneous vasculitis. There have also been several reported cases of systemic vasculitis following treatment with Adalimumab without mention of specific patient details or circumstances [1]. Anti-neutrophilic cytoplasmic antibody (ANCA) vasculitis has been rarely described.

\section{Case Presentation}

We present the case of a 57-year-old male with past medical history significant for coronary artery disease, hypertension and granuloma annulare (GA) who was admitted with rapid decline in renal function and shortness of breath. GA was being treated with adalimumab for the last two years. Eight months prior to admission, he was noted to have an asymptomatic elevation in his blood urea nitrogen and creatinine (Table 1) which worsened five months later. Renal ultrasound was performed which showed bilateral echogenic kidneys. He was lost to follow up and represented to his primary care provider three months later with a one-week history of epistaxis, hemoptysis, anorexia and weight loss. He was asked to report to the emergency room. 


\section{Cureus}

\begin{tabular}{|c|c|c|c|}
\hline \multirow[t]{2}{*}{ Laboratory Test } & Results & & \\
\hline & During hospitalization & Three months prior & Eight months prior \\
\hline Blood urea nitrogen $(7-18 \mathrm{mg} / \mathrm{dl})$ & 136 & 51 & 32 \\
\hline Creatinine $(0.55-1.3 \mathrm{mg} / \mathrm{dl})$ & 15.89 & 3.4 & 2.6 \\
\hline Hemoglobin (13.5-18 gm/dl) & 6.1 & & \\
\hline ANA Titre (Negative) & 1:80 (homogenous) & & \\
\hline p-ANCA Titre $(<1: 20)$ & $1: 40$ & & \\
\hline Proteinase 3 antibody ( $<1$ Al) & $<1$ & & \\
\hline Myeloperoxidase antibody (<1Al) & 5 & & \\
\hline c-ANCA (Negative) & Negative & & \\
\hline C3 complement (82-185 mg/dl) & 130 & & \\
\hline C4 complement (15-35 mg/dl) & 34 & & \\
\hline Hepatitis B surface antigen (Negative) & Negative & & \\
\hline Hepatitis C virus antibody (Negative) & Negative & & \\
\hline
\end{tabular}

\section{TABLE 1: Laboratory data on admission}

ANA: Anti-Nuclear Antibody; p-ANCA: perinuclear Anti-Neutrophilic Cytoplasmic Antibody; Al: Antibody Index; c-ANCA: cytoplasmic AntiNeutrophilic Cytoplasmic Antibody.

CT chest showed bilateral pulmonary consolidation and ground glass opacities (Figures 1,2). Renal biopsy performed revealed pauci-immune, rapidly progressive glomerulonephritis with some fibrosis (Figure 3). ANCA with perinuclear staining and myeloperoxidase antibody were positive. He was started on hemodialysis immediately. He also received intravenous methylprednisolone $500 \mathrm{mg}$ daily for three days followed by oral prednisone $60 \mathrm{mg}$ daily, oral cyclophosphamide $125 \mathrm{mg}$ daily (which was eventually transitioned to intravenous monthly pulses of cyclophosphamide) and trimethoprim-sulfamethoxazole for pneumocystis prophylaxis. Adalimumab was discontinued. 


\section{Cureus}

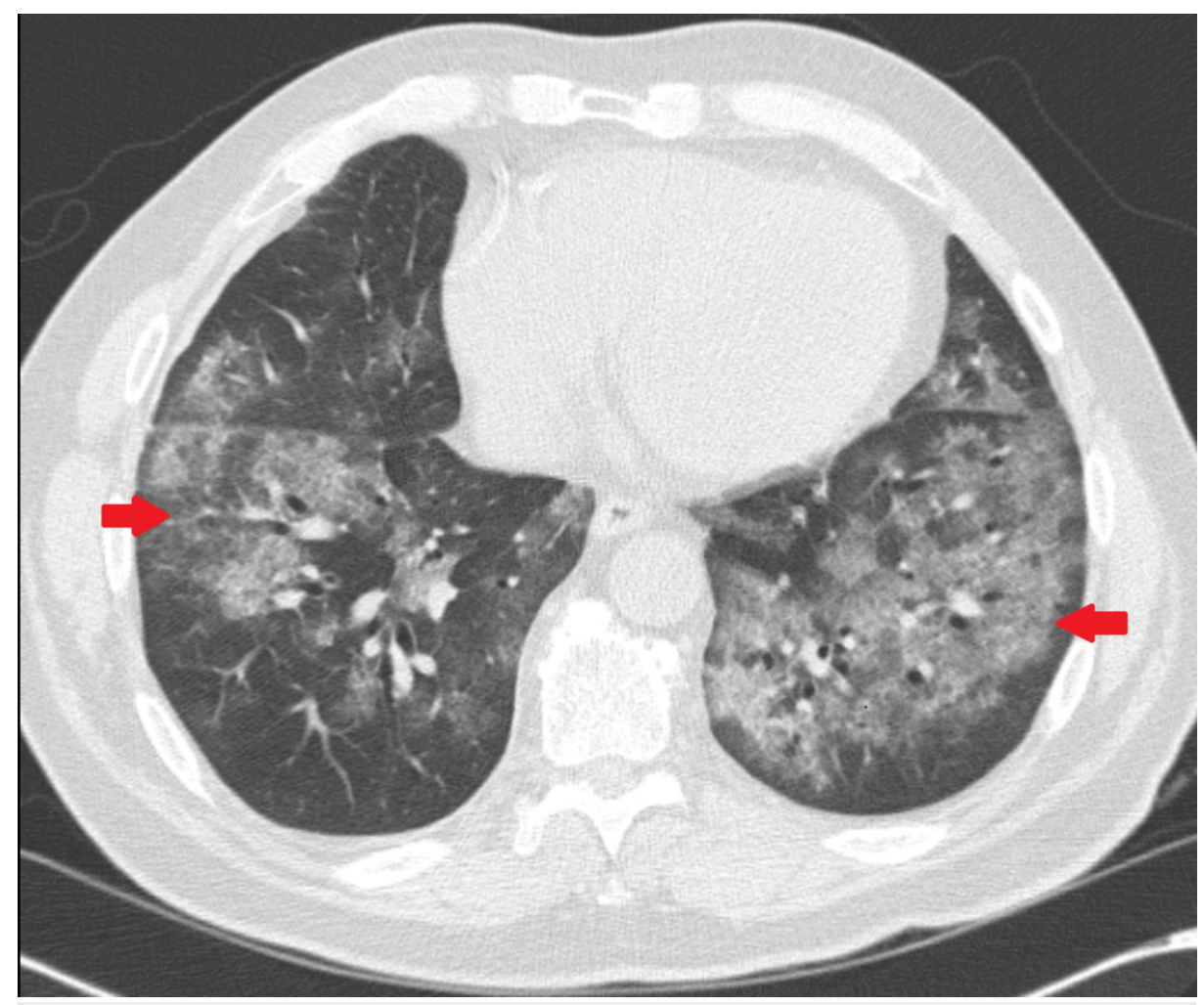

FIGURE 1: Axial CT chest image showing bilateral diffuse opacities (arrows)

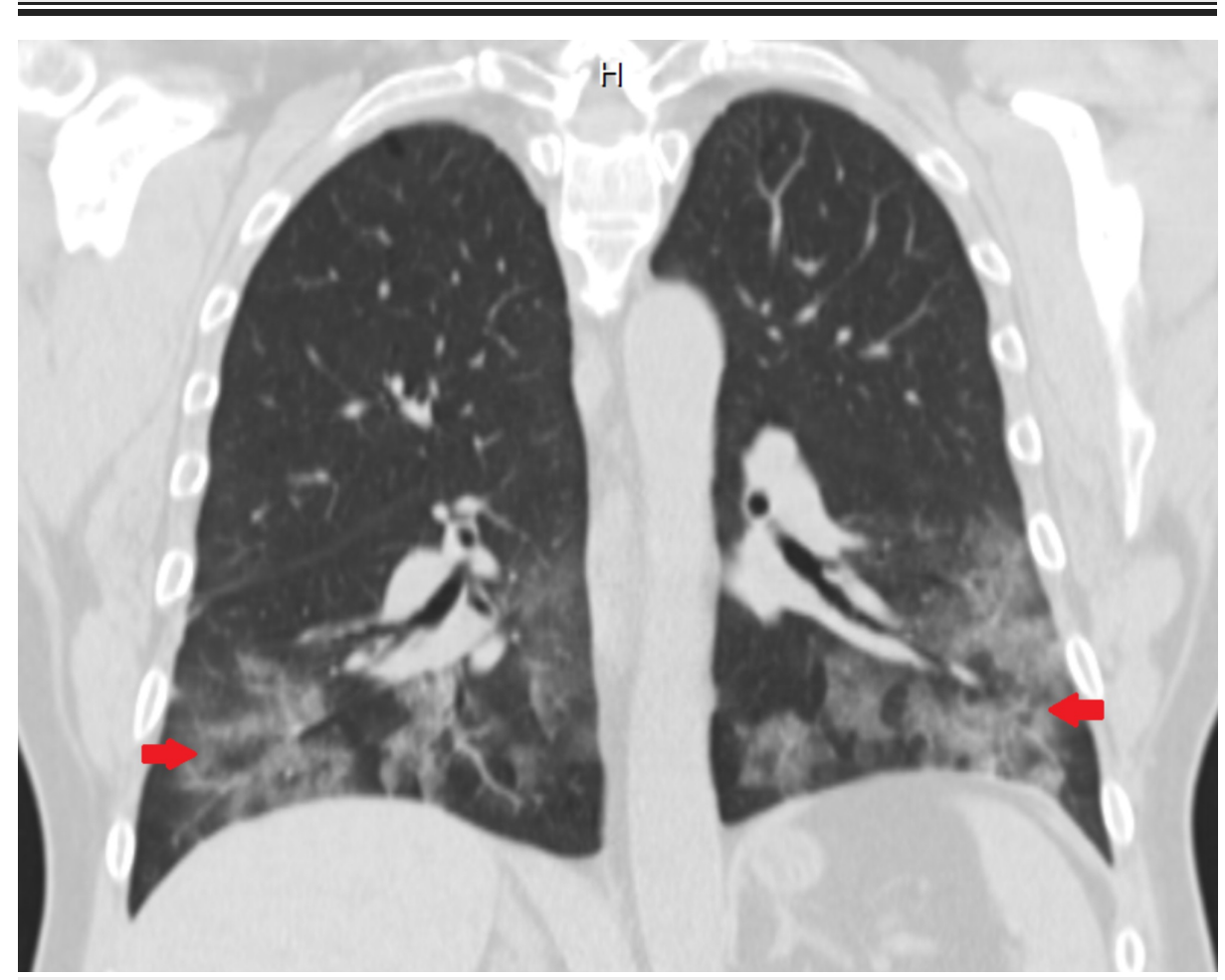

FIGURE 2: Coronal CT chest image showing bilateral basilar opacities (arrows) 


\section{Cureus}

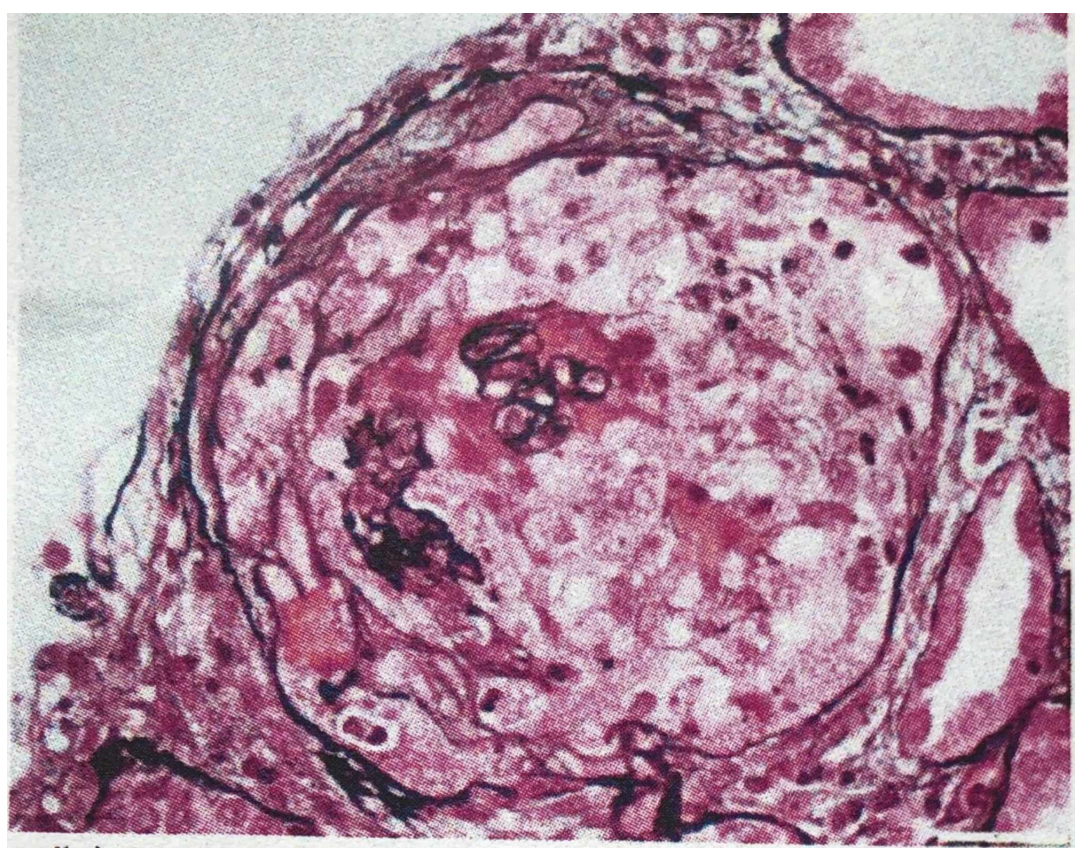

cellular crescent

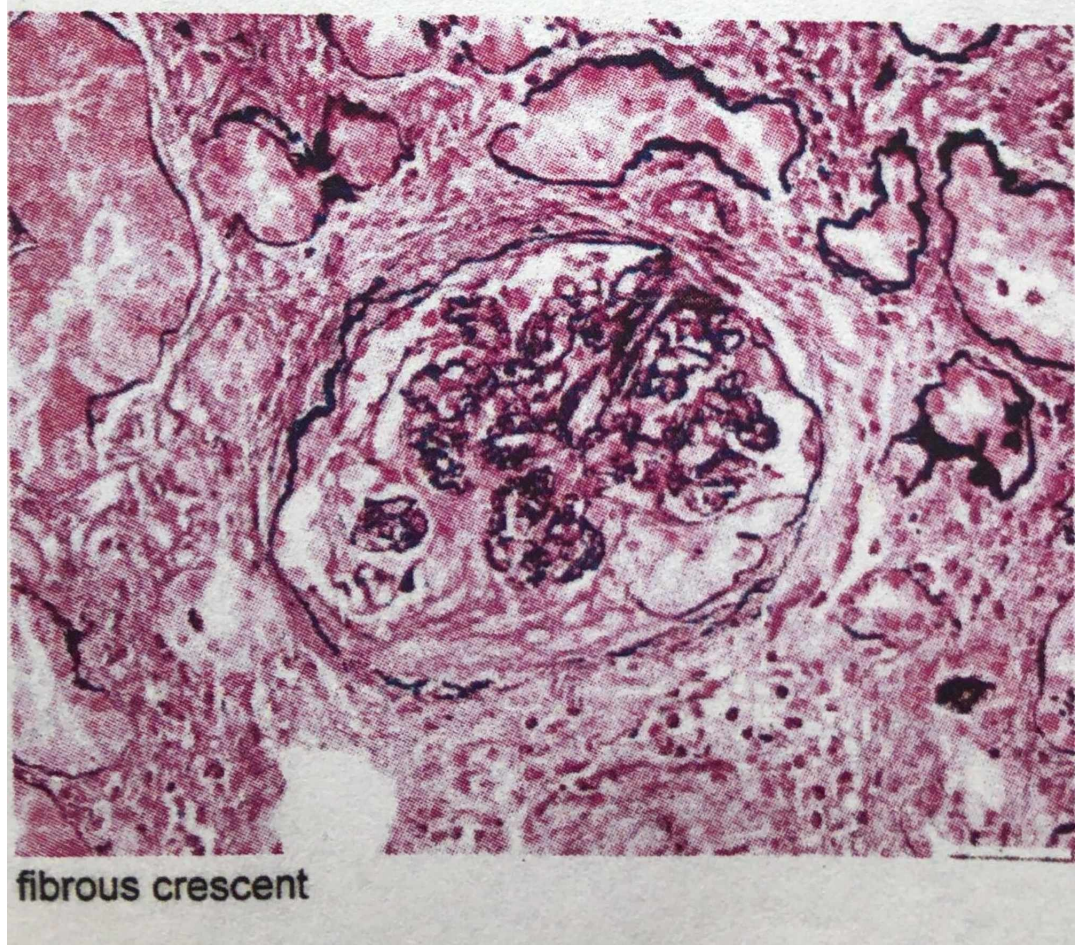

FIGURE 3: Renal biopsy images showing crescentic glomerulonephritis

Two months after his hospitalization, pulmonary infiltrates have resolved, but there has been no recovery of renal function.

\section{Discussion}

To our knowledge, there have only been nine previously reported cases of vasculitis and positive ANCA that were thought to be induced by TNF inhibitors [2-9]. See Table 2 for clinical presentation and treatment of each patient. One patient had atypical ANCA and lupus nephritis (Patient 8) and one patient had aortitis (Patient 9) which are not consistent with true ANCA-associated vasculitis. Of the remaining seven patients, four patients were females and six were being treated for rheumatoid arthritis. The mean age for patients was 51.4 years. Time of onset of symptoms after starting a TNF inhibitor varied from three months to four years. Four of these patients had positive c-ANCA, three had a positive p-ANCA. Six of seven patients had renal biopsies showing pauci-immune glomerulonephritis. Six patients were treated with intravenous 


\section{Cureus}

methylprednisolone followed by oral prednisone. The TNF inhibitor was discontinued in all cases except patient 7 . The most commonly used immunosuppressant was cyclophosphamide in six of seven patients. Four patients had persistent renal dysfunction and one patient died within nine months of presentation. Given the temporal sequence of events, a causal relationship might be present. One proposed mechanism is that anti-TNF drugs form immune complexes, activate complement and promote switching from a T-helper type 1 response (mediated by interleukin (IL)-1, TNF and interferon (IFN)-Y) to a T-helper type 2 response (IL-4, IL-5, IL-6, IL-10 and IL-13) leading to the production of autoantibodies [10].

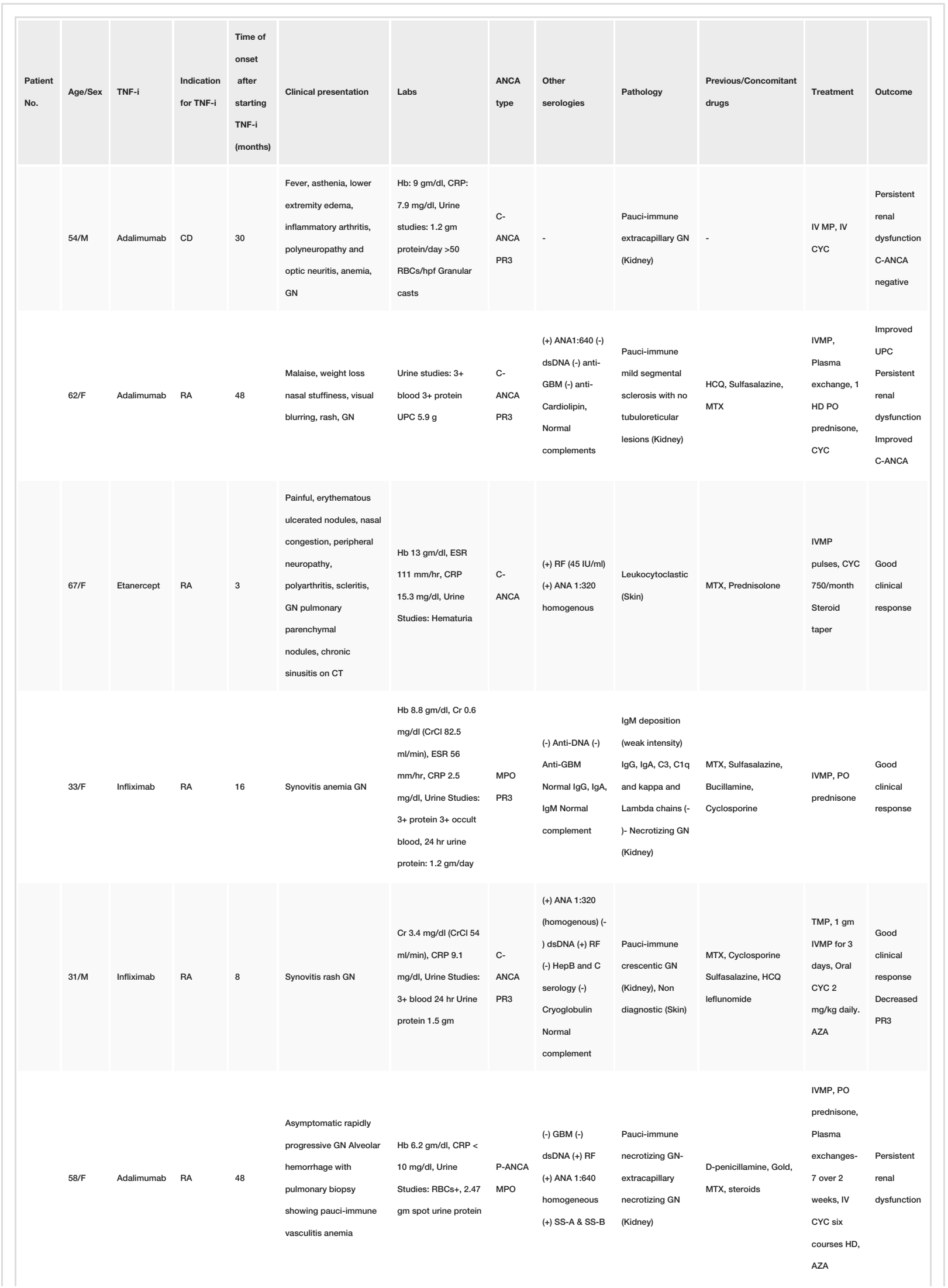




\section{Cureus}

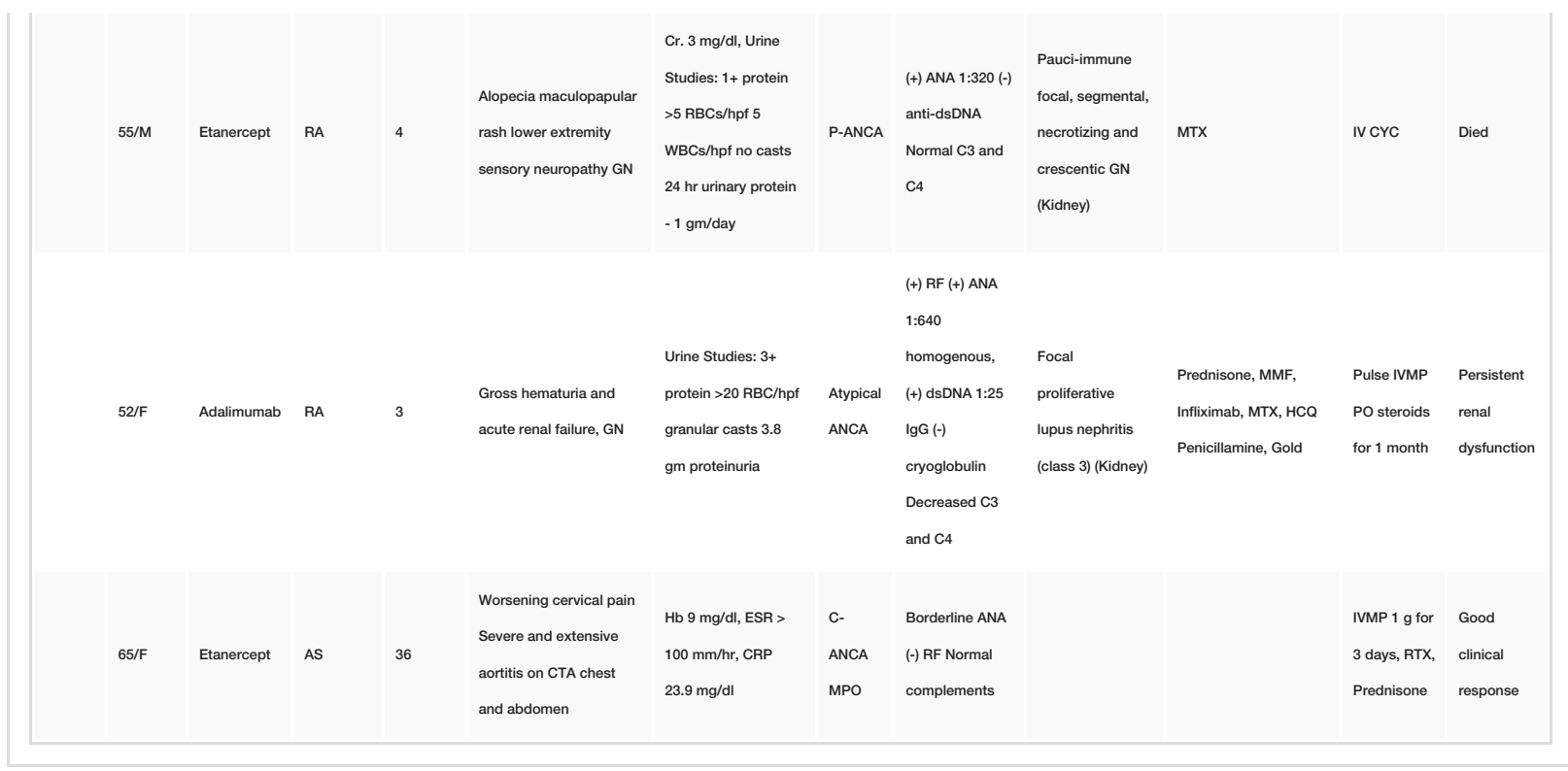

TABLE 2: Vasculitis with positive ANCA induced by TNF- inhibitors

ANCA: Anti-Neutrophilic Cytoplasmic Antibody; TNF-i: Tumor Necrosis Factor inhibitor; CD: Crohn's Disease; GN: Glomerulonephritis; Hb: Hemoglobin; CRP: C-Reactive Protein; RBC: Red Blood Cell; PR-3: Proteinase-3; IV: Intravenous; MP: Methylprednisolone; RA: Rheumatoid Arthritis; UPC: Urine Protein Creatinine; ANA: Anti-Nuclear Antibody; dsDNA: double stranded Deoxyribonucleic Acid; anti-GBM: anti-Glomerular Basement Membrane; HCQ: Hydroxychloroquine; MTX: Methotrexate; HD: Hemodialysis; ESR: Erythrocyte Sedimentation Rate; RF: Rheumatoid factor; MPO: Myeloperoxidase; SS: Sjogren's Syndrome; CrCl: Creatinine Clearance; PO: Per Oral; TMP: Trimethoprim; AZA: Azathioprine; RTX: Rituximab; Cr: Creatinine.

\section{Conclusions}

TNF-induced ANCA vasculitis is exceedingly rare. These cases in conjunction with ours suggest a possible relationship between anti-TNF use and induction of ANCA vasculitis. To the best of our knowledge, our case is the 4th to describe TNF-induced pulmonary renal syndrome as a manifestation of ANCA-vasculitis. It is difficult to conclusively prove that it is not just a spontaneous emergence of ANCA in a patient predisposed to autoimmunity. However, the biological plausibility of shifting towards a T-helper 2 type response in a susceptible individual leading to the emergence of these antibodies among others, remains. Additionally, this subset of patients appears to have a predilection for rapidly progressive kidney injury with long-term impairment despite discontinuation of the anti-TNF agent. This highlights the need for further studies looking into recognizing risk factors for the development of this rare but significant complication.

\section{Additional Information}

\section{Disclosures}

Human subjects: Consent was obtained by all participants in this study. Conflicts of interest: In compliance with the ICMJE uniform disclosure form, all authors declare the following: Payment/services info: All authors have declared that no financial support was received from any organization for the submitted work. Financial relationships: All authors have declared that they have no financial relationships at present or within the previous three years with any organizations that might have an interest in the submitted work. Other relationships: All authors have declared that there are no other relationships or activities that could appear to have influenced the submitted work.

\section{References}

1. Ramos-Casals M, Brito-Zeron P, Soto MJ, Cuadrado MJ, Khamashta MA: Autoimmune diseases induced by TNF-targeted therapies: analysis of 233 cases. Medicine. 2007, 86:242-251. 10.1097/MD.0b013e3181441a68

2. Fournier A, Nony A, Rifard K: Antineutrophil cytoplasmic antibody associated vasculitis in a patient treated with adalimumab for a rheumatoid arthritis. Nephrol Ther. 2009, 5:652-657. 10.1016/j.nephro.2009.03.005

3. Martin Varas C, Heras M, Saiz A, et al.: Antineutrophil cytoplasmic antibodies associated vasculitis in patient with Crohn's disease treated with adalimumab. Nefrologia. 2017, 37:560-561. 10.1016/j.nefro.2017.03.009

4. Simms R, Kipgen D, Dahill S, Marshall D, Rodger RS: ANCA-associated renal vasculitis following anti-tumor necrosis factor alpha therapy. Am J Kidney Dis. 2008, 51:11-14. 10.1053/j.ajkd.2007.10.043

5. Stokes MB, Foster K, Markowitz GS, et al.: Development of glomerulonephritis during anti-TNF-alpha therapy for rheumatoid arthritis. Nephrol Dial Transplant. 2005, 20:1400-1406. 10.1093/ndt/gfh832

6. Hirohama D, Hoshino J, Hasegawa E, et al.: Development of myeloperoxidase-antineutrophil cytoplasmic 


\section{Cureus}

antibody-associated renal vasculitis in a patient receiving treatment with anti-tumor necrosis factor-alpha. Mod Rheumatol. 2010, 20:602-605. 10.1007/s10165-010-0339-x

7. Ortiz-Sierra MC, Echeverri AF, Tobon GJ, Canas CA: Developing of granulomatosis with polyangiitis during etanercept therapy. Case Rep Rheumatol. 2014, 2014:3. 10.1155/2014/210108

8. Ashok D, Dubey S, Tomlinson I: C-ANCA positive systemic vasculitis in a patient with rheumatoid arthritis treated with infliximab. Clin Rheumatol. 2008, 27:261-264. 10.1007/s10067-007-0712-0

9. Ginsberg S, Rosner I, Slobodin G, et al.: Etanercept treatment-related c-ANCA-associated large vessel vasculitis. Clin Rheumatol. 2016, 35:271-273. 10.1007/s10067-015-3134-4

10. Guillevin L, Mouthon L: Tumor necrosis factor-alpha blockade and the risk of vasculitis . J Rheumatol. 2004, 31:1885-1887. 